\title{
ELABORACIÓN DE LA FIBRA DE CABUYA EN TEJIDO PLANO COMO MATRIZ DE REFUERZO PARA LA CONSTRUCCIÓN DE UN RETROVISOR
}

\author{
ELABORATION OF THE CABUYA FIBER IN FLAT \\ FABRIC AS REINFORCEMENT MATRIX FOR THE \\ CONSTRUCTION OF A REAR-VIEW MIRROR
}

\author{
Luis Pruna $^{1, *}$, Fabián Velasco ${ }^{1}$, Fabián Chachapoya ${ }^{1}$, Cristian Paredes ${ }^{1}$
}

\section{Resumen}

Las fibras naturales se han convirtiendo en una excelente alternativa para usos industriales. Esto se debe a su fácil accesibilidad en el mercado y por ser una materia prima renovable. El presente trabajo busca usar la cabuya como material de refuerzo con matriz poliéster para aplicaciones automotrices con bajo costo y peso. Este es el caso de los retrovisores de un vehículo, para el cual se fabricó un molde base. Sobre el molde se realizará el aplicado y moldeado del retrovisor utilizando la fibra de cabuya y una resina poliéster. Las mezclas de octoato de cobalto y peróxido de metil-etilcetona (MEKP) con la fibra natural reducen considerablemente el peso; el costo de manufactura se ve reducido aproximadamente en un $40 \%$ debido a la facilidad de manejo de la fibra y la adquisición de este material. Se recomienda la utilización de la cabuya para aplicaciones automotrices (espejo retrovisor) ya que presenta una reducción considerable en su peso y costo relativamente bajo en comparación con el componente original.

Palabras clave: aplicaciones, fibra natural, extracción, moldeado, proceso.

\begin{abstract}
Natural fibers are becoming an efficient alternative for industrial applications. This is due to its easy accessibility in the market and for being a renewable raw material. The present work seeks to replace the cabuya as reinforcement material with polyester matrix for automotive applications with low cost and weight. This is the case of the mirrors of a vehicle, for which a base mold was manufactured. On the mold will be applied and molded the mirror on which the use of the fiber of cabuya and a polyester resin is detached. Mixtures of cobalt octoate and methyl ethyl ketone peroxide (MEKP) with natural fiber significantly reduce weight; the manufacturing cost is reduced by approximately $40 \%$ due to the ease of handling of the fiber and the acquisition of this material. The application of the cabuya is recommended for automotive applications such as the rearview mirror as it presents a considerable reduction in its weight and relatively low cost compared to the original component.
\end{abstract}

Keywords: Applications, natural fiber, extraction, molding, process.

\footnotetext{
${ }_{1, *}$ Departamento de Ciencias de la Energía y Mecánica. Universidad de las Fuerzas Armadas ESPE, Latacunga, Ecuador. Autor para correspondencia : lrpruna@espe.edu.ec. (D) http://orcid.org/0000-0002-0651-9597,

(D) http://orcid.org/0000-0003-0609-3853, (D) http://orcid.org/0000-0001-7015-664X,

(D) http://orcid.org/0000-0001-5708-5883
}

Recibido: 21-02-2020, aprobado tras revisión: 03-06-2020

Forma sugerida de citación: Pruna, L.; Velasco, F.; Chachapoya. F. y Paredes, C. (2020). «Elaboración de la fibra de cabuya en tejido plano como matriz de refuerzo para la construcción de un retrovisor». InGENIUS. N. ${ }^{\circ} 24,($ julio-diciembre). pp. 81-86. DOI: https://doi.org/10.17163/ings.n24.2020.08. 


\section{Introducción}

Las fibras naturales son estructuras unidimensionales, largas y delgadas. Se doblan con facilidad y su propósito principal es la creación de tejidos. Su clasificación se basa según su origen vegetal, animal o mineral. A su vez, las fibras de origen vegetal se clasifican de acuerdo con la parte de la planta de la que se extraen [1], esto permite obtener una mejor nomenclatura de las fibras obtenidas con nuevas investigaciones.

La cabuya es una planta muy abundante en la región central del Ecuador, donde es usada por los campesinos para alimentar al ganado vacuno. La fibra se obtiene por medio de un procesamiento por etapas: despenque, machacado, cocción, secado, claseado.

En el Ecuador, el desarrollo de materiales compuestos se encuentra en sus etapas iniciales, por ende, el uso de fibras naturales todavía es limitado. En general, la extracción de fibras vegetales se realiza manualmente, lo que puede cambiar tomando en cuenta el gran potencial que el país ofrece. Resulta importante conocer la producción de fibras naturales nacionales [2].

Por otro lado, la necesidad de materiales más amigables con el medioambiente ha impulsado el estudio de los polímeros naturales para su empleo en aplicaciones desechables a fin de contar con un material disponible en la naturaleza y que, a la vez, sea biodegradable [3].

Estos materiales compuestos representan una importante alternativa para el reemplazo de los compuestos reforzados a base de metal, aluminio, cromo, tungsteno, etc.; sus propiedades resultan en muchas ocasiones comparables e incluso superiores.

La reducción de peso del vehículo se ha considerado como una de las soluciones más importantes para mejorar el ahorro de combustible, la reducción pesopotencia y llegar a tener pocas o nulas emisiones contaminantes. Se cree que el peso del cuerpo del vehículo se puede reducir con el uso de múltiples materiales y sin aumento de costo [4].

De la gran variedad de materiales compuestos que existen en el mercado, se describe que están formados por matrices orgánicas (epoxi, viniléster, poliéster) y fibras de alta resistencia (vidrio, carbono, fibras naturales, etc.), considerándose a estos como los más desarrollados y utilizados a nivel industrial [5].

Con respecto a las fibras, estas son materiales policristalinos o amorfos, tienen diámetros pequeños y poseen gran longitud. Los materiales de las fibras son generalmente polímeros o cerámicos (por ejemplo, aramidas, vidrio, carbono, boro, óxido de aluminio y carburo de silicio). También se tiene la utilización de fibras naturales como abacá, cabuya y coco, incorporados como elementos de refuerzo en una matriz polimérica. Este tipo de materiales ofrecen muchas ventajas, entre las cuales cabe resaltar la reducción de costo de manufactura y su menor impacto ambiental [6].
Desde siempre en las competencias automovilísticas, los vehículos que participan poseen un peso considerablemente bajo, como es el caso de la Fórmula 1 , permitiendo que estos tengan un gran rendimiento, porque usan materiales más ligeros en la elaboración de sus componentes, y con características similares a originales.

En el Ecuador (2008), se produjo aproximadamente 5400 toneladas de fibra de cabuya, según el Ministerio de Agricultura, Ganadería, Acuacultura y Pesca (MAGAP). Las industrias ecuatorianas empezaron a realizar pruebas para conocer la resistencia de esta fibra [6], y con ello sus posibles aplicaciones y el desarrollo de nuevos materiales compuestos biodegradables.

El objetivo de usar fibras naturales en la elaboración de un retrovisor es facilitar que este elemento pueda llegar a ser reciclado, contribuyendo a reducir el impacto ambiental causado por la industria automotriz por el uso de varios materiales en la elaboración de los automóviles, como es el caso de los plásticos, y al mismo tiempo reducir la masa de sus componentes [7], [8].

Se espera la obtención de un retrovisor más ligero, con una considerable resistencia a las deformaciones a causa de impactos, así como una apreciable reducción de su costo de manufacturación, teniendo en cuenta un criterio de sustentabilidad en cada proceso de elaboración; también la posibilidad de introducir la fibra de cabuya en la industria automotriz del país, permitiendo el desarrollo de nuevas aplicaciones [9-11].

\section{Materiales y métodos}

\subsection{Extracción de la fibra de cabuya}

Es necesaria una correcta selección de la planta de cabuya para contar con pencas de mayor tamaño que, a su vez, permitan obtener más fibra con menos plantas, con lo cual mejoramos la eficiencia en la producción. El tiempo aproximado de crecimiento es de 5 años antes de ser despencada utilizando una barra de acero con punta filosa.

Para extraer la penca se deben seguir los siguientes pasos:

1. Cortar con un machete la penca que se halle más cercana al suelo.

2. Picar la planta con la ayuda de la barra en el sitio de la penca cortada (Figura 1), hasta llegar al centro, una vez logrado eso se debe aplicar una fuerza perpendicular sobre la barra para que la planta se abra (es decir, que se levante), así todas las pencas quedan libres.

3. Seguidamente se tiene que quitar con el machete las espinas que tiene cada penca.

4. Con la mano se toma la punta de la penca y la halamos hacia nosotros realizando un movimiento 
torsor hasta desprenderla de su centro; esto se conoce como llagui.

5. Repetir el paso 4 con todas las pencas.

6. Una vez que se extraen todas se las traslada al área de extracción de la fibra.

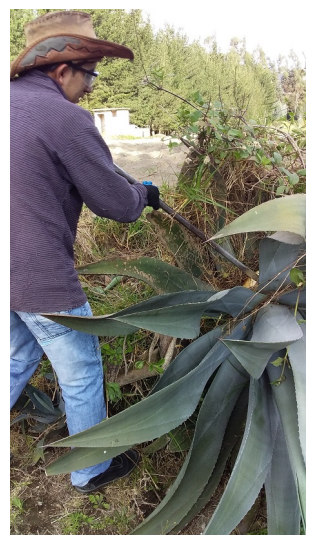

Figura 1. Extracción de la fibra

Para obtener la fibra, con la ayuda de un mazo sobre una superficie plana, se procede a machacar las pencas para extraer toda el agua que tienen en su interior. Luego con un machete se quita la capa verdosa que cubre a la penca. Con la ayuda de una tabla de madera se realizan movimientos hacia arriba y hacia abajo de la superficie de la penca para extraer los residuos de agua y la capa protectora, hasta obtener la fibra deseada, que puede presentar un color blanco verdoso en ciertas zonas, ver la Figura 2.

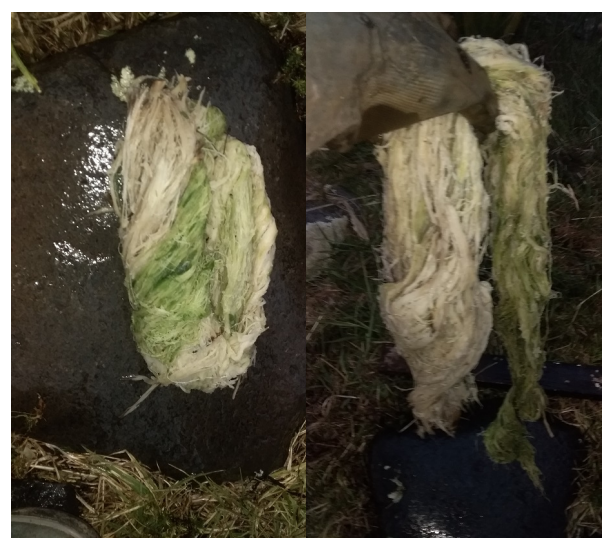

Figura 2. Fibra en bruto

Una vez que se tiene la fibra se procede a su cocción por unos 100 minutos; la misma adquiere un color blanco intenso. Se la pone a secar por cuatro días en un alambre, con la ayuda de pinzas se la fija. Es importante que la fibra no se moje al estar colgada porque puede perjudicarse su calidad (Figura 3). Cuando la fibra está completamente seca presenta un color amarillo pálido; se continúa con su peinado (claseado) utilizando una tabla con clavos para separar las fibras más pequeñas, dando como resultado una fibra uniforme, lista para usar.

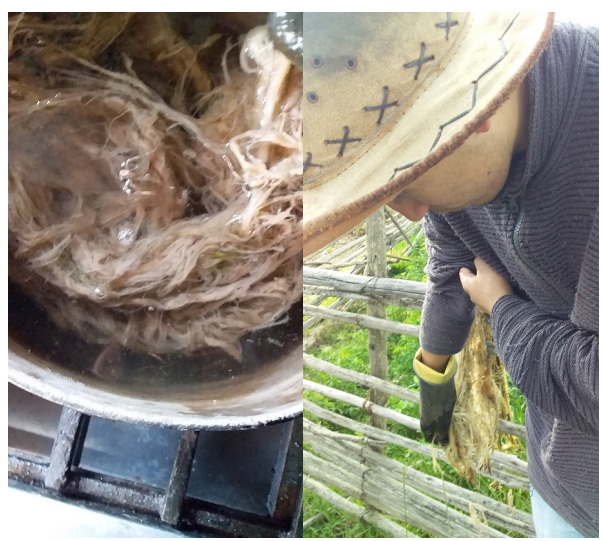

Figura 3. Cocinado y secado de la fibra

\subsection{Tejido de la fibra de cabuya}

Para ello se usa un tablero cuadriculado de madera, para tener la máxima precisión en la colocación de las hebras de la fibra. El tejido usado para este caso fue un tramado a 90 grados: se coloca la fibra de cabuya en forma horizontal a cada $2 \mathrm{~mm}$ de espaciado y en lo que se refiere a la parte vertical se realiza a cada 10 mm de espaciado; cada uno de los conjuntos de hebras colocadas debe tener un total de 4 hebras (Figura 4). El tejido final tiene $40 \mathrm{~cm}$ de ancho x $30 \mathrm{~cm}$ de alto.

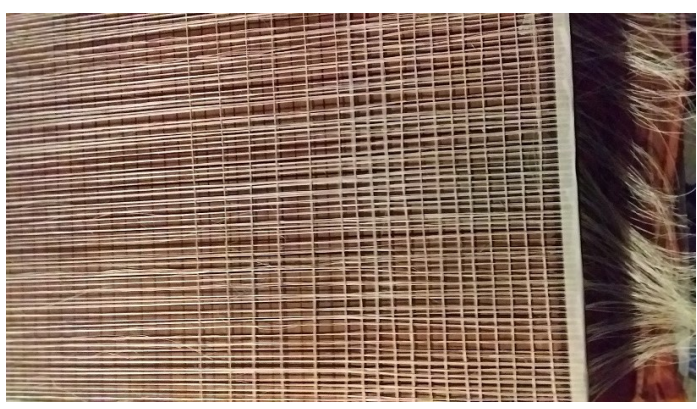

Figura 4. Tejido de la fibra

Se procede a amarrar los extremos de cada grupo de hebras para que el tejido no tienda a deformarse en la posterior manipulación.

\subsection{Proceso de fabricación del retrovisor}

Para la fabricación del retrovisor se sigue el siguiente proceso:

1. Almacenamiento de la resina y fibra correspondiente.

2. Preparación de la fibra.

3. Preparación del molde. 
4. Preparación de la mezcla.

5. Colocación de la matriz (cabuya).

6. Solidificación.

7. Desmoldar.

8. Lijado.

9. Inspección de acabado.

10. Verificación de pesos.

Al retrovisor original se le hace una limpieza integral, luego viene el despiece del espejo así como de los demás accesorios (Figura 5).

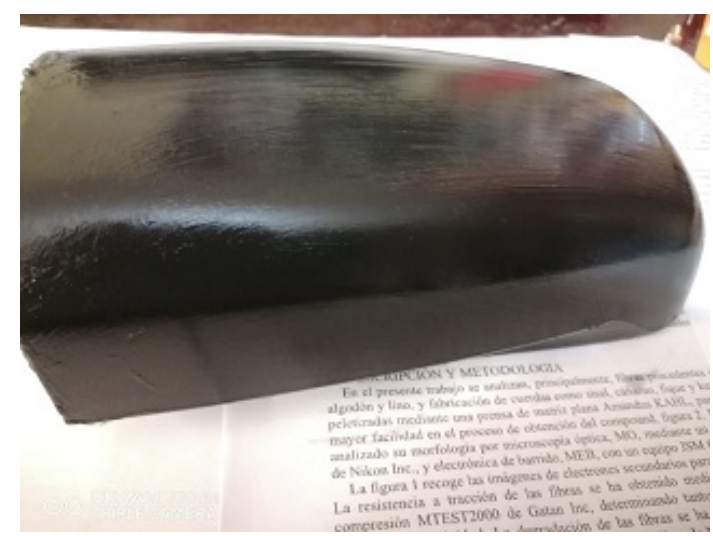

Figura 5. Limpieza de impurezas del retrovisor original

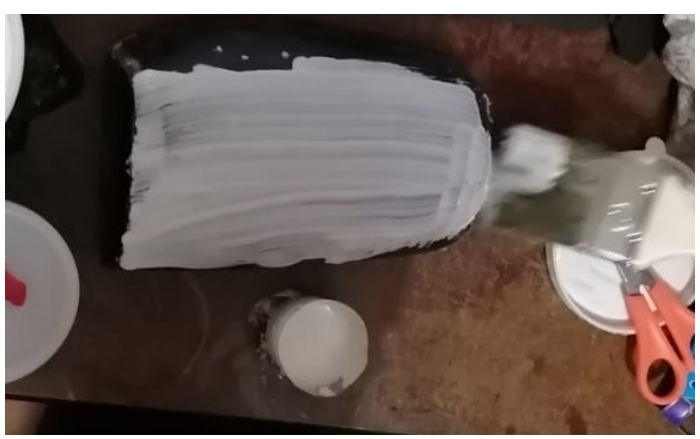

Figura 6. Aplicación de la cera desmoldante

Cuando se tiene el molde limpio de impurezas se procede a la colocación de la cera desmoldante tal y como se muestra en la Figura 6, con la finalidad que se pueda retirar el retrovisor al finalizar el secado.

Como matriz se utiliza la fibra de cabuya; para el curado de la resina el octoato de cobalto y el peróxido de metil-etilcetona (MEKP) o catalizador, como refuerzo se trabaja con dos capas de fibra de cabuya (Figura 7).

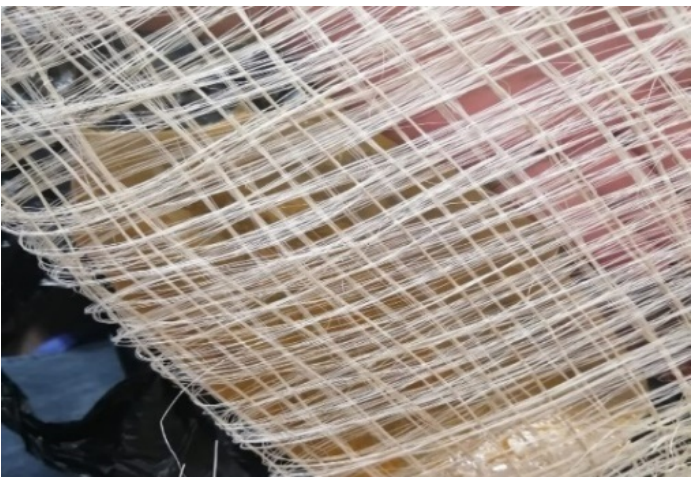

Figura 7. Fibra de cabuya elaborada y tejida a mano

Las proporciones de la resina, del octoato de cobalto y del catalizador deben ser exactas, pues deben cumplir con la composición marcada en la Tabla 1. Para la aplicación de la mezcla se emplea una brocha teniendo cuidado de hacerlo de forma uniforme para evitar la acumulación en ciertas áreas.

Tabla 1. Cantidades de resina, octoato de cobalto, MEKP y fibra de cabuya, utilizadas para el material compuesto del retrovisor

\begin{tabular}{cc}
\hline Descripción & Cantidad \\
\hline Espesor [mm] & 2,5 \\
Número de capas de resina & 2 \\
Fibra de cabuya tejida [g] & 25 \\
Resina poliéster [ml] & 150 \\
Octoato de cobalto [ml] & 0,75 \\
MEKP [ml] & 0,4 \\
\hline
\end{tabular}

En la tabla anterior se especifican los parámetros con los cuales se va a hacer el retrovisor, así como la cantidad de los reactivos químicos necesarios para una mayor velocidad de secado de la resina. Se deja secar cada parte del retrovisor por tres días para conseguir una mejor consistencia y dureza. Cumpliendo con estas recomendaciones se obtuvo un retrovisor con mejores características de resistencia y una considerable reducción de peso (Figura 8).

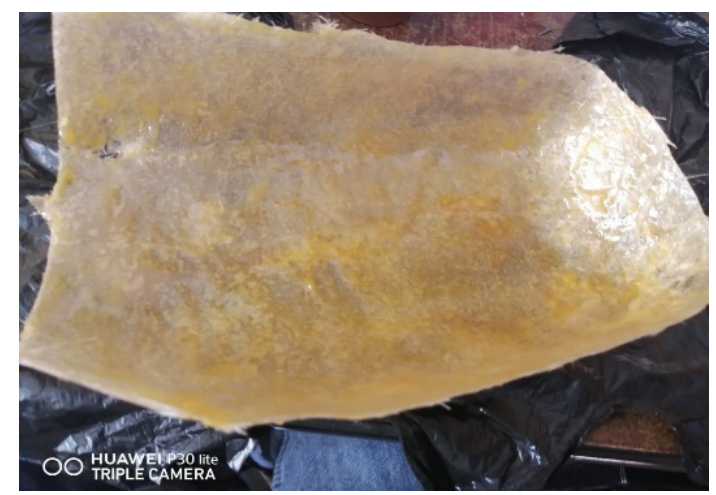

Figura 8. Retrovisor con una matriz de fibra de cabuya 


\subsection{Diseño del mecanismo para el giro en $180^{\circ}$}

Para el mecanismo se toma como base una bisagra que se abre y cierra continuamente como la que usamos en la puerta de nuestros hogares, porque el retrovisor debe mantener su posición inicial y recuperarse una vez que soporte un impacto y haya sido movido un cierto grado desde su origen. Para esto se decide colocar a los lados del retrovisor unos resortes de las mismas características, con esto se consigue que si se mueve el retrovisor a un lado uno de los resortes se comprime y el otro se extiende y una vez que se deje de aplicar la fuerza, se regresa al estado inicial.

El modelado del mecanismo se lo realiza en $\mathrm{Au}$ todesk Inventor (Figura 9); se deben analizar los principales puntos sobre los cuales se va a ejercer un mayor esfuerzo al aplicar una fuerza sobre el mecanismo. Con esta información se toman las precauciones necesarias en su elaboración para evitar posibles fallas durante su funcionamiento (quizás sea necesario reforzar ciertas zonas).

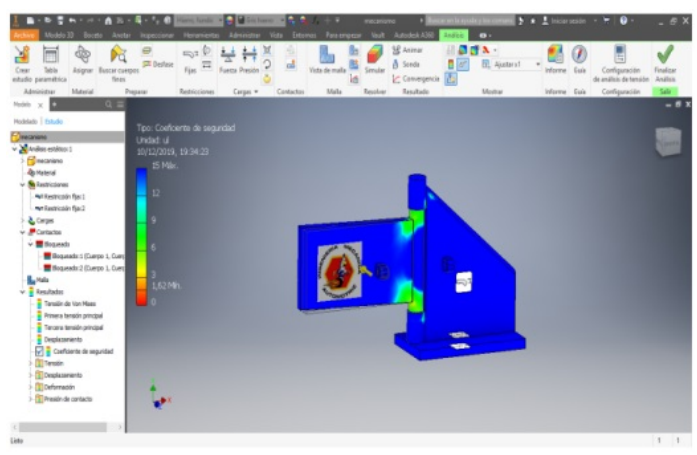

(a)

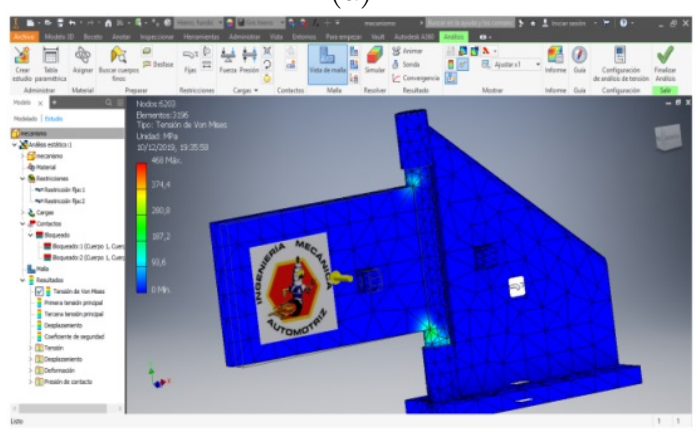

(b)

Figura 9. Análisis del mecanismo en Autodesk Inventor. a) Coeficiente de seguridad. b) Tensión de Van Mises

En la Figura 10 se observan los resultados del análisis del retrovisor, el mismo que debe cumplir lo dispuesto en la Norma NTE INEN 1323.

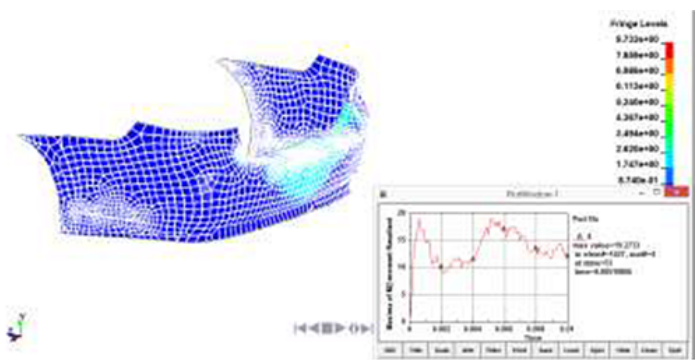

Figura 10. Análisis del retrovisor usando el software Ansys

\section{Resultados y discusión}

En la Tabla 2 se resumen las comparaciones de pesos obtenidos al aplicar la fibra de cabuya en la realización del retrovisor, lo cual se puede traducir como un total éxito dado que las mediciones obtenidas nos muestran una reducción considerable de su peso, con lo cual se ayuda a la reducción de peso de los automóviles.

Tabla 2. Comprobación de los pesos

\begin{tabular}{cc}
\hline Descripción & Peso (kg) \\
\hline Con la fibra de cabuya con el mecanismo & 0,223 \\
Con la fibra de cabuya sin el mecanismo & 0,1859 \\
Retrovisor original & 0,227 \\
\hline
\end{tabular}

En la Tabla 3 se plasma el comportamiento que llegó a tener el retrovisor de fibra de cabuya a la aplicación de una fuerza en su superficie, con lo cual se verificó la resistencia del mecanismo de giro de $180^{\circ}$; se observó que los soportes de los resortes de recuperación deben ser reforzados para evitar la fractura de estos pequeños alojamientos del resorte; de igual forma se pudo visualizar que al aplicar una mayor fuerza sobre la superficie del retrovisor esta tiende a descascararse en una pequeña zona; el área total del retrovisor es aproximadamente de $150 \mathrm{~mm}^{2}$ en la cual se va a realizar el análisis de resistencia.

Tabla 3. Resistencia al impacto

\begin{tabular}{ccc}
\hline & $\begin{array}{c}\text { Fuerza/área } \\
(\mathbf{M P a})\end{array}$ & Fuerza (N) \\
\hline Primera prueba & 0,039 & 5,86 \\
Segunda prueba & 0,019 & 2,9 \\
Tercera prueba & 0,01 & 1,45 \\
\hline
\end{tabular}

Luego de realizar el análisis de cada una de las pruebas, se puede determinar que la fibra de cabuya y todas las fibras naturales presentan una baja resistencia mecánica, por tal motivo el accesorio que se desee construir no debe estar sometido a cargas excesivas. 
La fibra de cabuya tiene una apariencia flexible, su manipulación es sencilla y no se fractura con facilidad. En cambio, otras fibras son bastante frágiles, se rompen con facilidad y son rígidas, lo cual dificulta su manipulación. En el caso del bagazo de caña la longitud de sus fibras es menor que las de los otros especímenes, lo cual hace más compleja su aplicación en la industria automotriz; además, estas fibras presentan residuos adheridos que son los conductos en donde se almacena la sacarosa, generando que estas sean de estructura menos homogénea.

\section{Conclusiones}

La mezcla de octoato de cobalto y peróxido de metil-etilcetona (MEKP) con la fibra natural de cabuya tiende a reducir considerablemente el peso del retrovisor y aumentar su dureza en relación con el de fabricación tradicional.

El costo de manufactura se reduce al realizarlo con esta fibra, casi en un $40 \%$.

Debido a la facilidad de manejo de la fibra y la adquisición de materiales a utilizar la estratificación manual es uno de los procesos más empleados para la elaboración de equipos en la industria automotriz.

La selección del material o de la fibra natural a utilizar dependerá de los requerimientos propios de la aplicación a realizar.

Los resultados son alentadores para tratar de impulsar el uso de las fibras naturales nacionales en el desarrollo de otros elementos (piezas) automotrices, ayudando al cuidado del ambiente al obtener productos biodegradables.

\section{Referencias}

[1] S. M. Velásquez Restrepo, G. J. Pelaéz Arroyave, and D. H. Giraldo Vásquez, "Uso de fibras vegetales en materiales compuestos de matriz polimérica: una revisión con miras a su aplicación en el diseño de nuevos productos," Informador Técnico, vol. 80, no. 1, pp. 77-86, jun. 2016. [Online]. Available: https://doi.org/10.23850/22565035.324

[2] D. J. Moreano Moreano and D. A. Zambrano Romero, "Diseño y construcción de parachoques delantero y posterior de un vehículo chevrolet optra año 2008 a partir de fibra natural de la planta de abacá," 2016. [Online]. Available: https://bit.ly/2BuYpid

[3] O. A. Jiménez Arévalo, M. A. Sánchez-Soto, and M. Trujillo Barragán, "Impacto de baja energía en un compuesto almidón-fibra natural," in $X V$ Congreso Internacional anual de la SOMIM Sonora. México, 2009, pp. 690-695. [Online]. Available: https://bit.ly/3eMDPrO

[4] H. Iza, L. Quiroz, and F. Salazar, "Aplicación de fibra de yute en la construcción de carrocerías para vehículos fórmula SAE," Revista: Energía y Mecánica, Innovación y Futuro, vol. 1, no. 4, pp. 102-111, 2015. [Online]. Available: https://bit.ly/36YdiFx

[5] A. Morales, D. Valenzuela, T. Lozano, and M. Ponce, "Materiales reforzados de poliolefinas recicladas y nanofibras de celulosa de henequén," Revista Iberoamericana de Polímeros, vol. 12, no. 5, pp. 255-267, 2011. [Online]. Available: https://bit.ly/2Y1sayy

[6] V. Guerrero, J. Dávila, S. Galeas, P. Pontón, N. Rosas, V. Sotomayor, and D. Valdivieso, Nuevos materiales: Aplicaciones estructurales e industriales. Imprefep, 2011. [Online]. Available: https://bit.ly/3gU6Tjg

[7] S. Aguilar, J. Ramírez, and O. Malagón, "Extracción de fibras no leñosas Cabuya (Furcraea andina Trel.) y banano (Musa Paradisiaca L.) para estandarizar un proceso tecnológico destinado a la elaboración de pulpa y papel," Revista Iberoamericana de Polímeros, vol. 8, no. 2, pp. 89-98, 2007. [Online]. Available: https://bit.ly/2XW6LHh

[8] H. G. Villacís Salazar, "Obtención de materiales compuestos híbridos de matriz poliéster reforzados con fibra de vidrio y abacá mediante estratificación," 2011. [Online]. Available: https://bit.ly/306jQjF

[9] S. Kalia, B. S. Kaith, and I. Kaur, Cellulose Fibers: Bio-and Nano-Polymer Composites. Springer, Berlin, Heidelberg, 2011. [Online]. Available: https://doi.org/10.1007/978-3-642-17370-7

[10] F. R. Delgado Arcentales, S. G. Galeas Hurtado, and V. H. Guerrero Barragán, "Obtención de materiales compuestos híbridos de matriz poliéster reforzada con fibra de coco y de vidrio para la elaboración de tableros," Revista Politécnica, vol. 33, no. 1, 2014. [Online]. Available: https://bit.ly/3gMfO66

[11] A. Mohanty, M. Misra, and L. Drzal, Natural fibers, biopolimers and biocomposites. CRC Press, 2005. [Online]. Available: https://bit.ly/3gPyzpk 